\title{
TIPOLOGIA DOS MUNICÍPIOS DO ESTADO DE MINAS GERAIS EM FUNÇÃO DA COMPENSAÇÃO POR SERVIÇOS AMBIENTAIS E DA PRODUÇÃO AGRÍCOLA
}

Rodrigo Pinheiro Ribas ${ }^{1}$

\section{Resumo}

A compensação por serviços ambientais caracteriza-se como a transferência de recursos para aqueles que se dedicam à prática de conservação da natureza. O ICMS Ecológico é uma destas formas de repasse de recursos aos municípios segundo critérios ambientais.O objetivo deste artigo é traçar um perfil dos municípios do Estado de Minas Gerais que receberam o ICMS Ecológico no ano de 2010 em função da sua produção agrícola no mesmo. Com isso pretende-se realizar uma análise dos agrupamentos formados por meio da técnica Two Step Cluster, oferecendo subsídio para o entendimento da configuração espacial do pagamento por serviços ambientais em Minas Gerais e o seu relacionamento com a atividade agrícola. Foram identificados três clusters, sendo um de grandes produtores agrícolas que recebem grandes valores de repasse do ICMS Ecológico, outro cluster que caracteriza-se por não receber repasses de ICMS Ecológico e baixa produção agrícola e, por fim, um cluster com municípios recebendo valores de repasses de ICMS Ecológico acima da média, porém com baixos valores relacionados à produção agrícola.

Palavras-chaves:ICMS Ecológico, Análise de Agrupamentos, Atividade Agrícola

\begin{abstract}
Compensation for environmental services is characterized as the transfer of resources for those engaged in the practice of nature conservation. The Ecological VAT is one of these forms of funds transfer to municipalities according to environmental criteria. The purpose of this article is to draw a profile of the municipalities of Minas Gerais who received the Ecological VAT in 2010 according to their agricultural production. We intend to conduct an analysis of the groups formed through technical Two Step Cluster, providing subsidy for understanding the spatial configuration of the payment for environmental services in Minas Gerais and their relationship with agriculture. Three clusters were identified, large agricultural producers who receive large transfer values of the Ecological VAT, another cluster that is characterized by not receive Ecological VAT transfers and low agricultural production and, finally, a cluster with municipalities receiving transfers of Ecological VAT above average, but with low values related to agricultural production.
\end{abstract}

Keywords:Ecological VAT, Cluster Analysis, Agricultural Activity

1Universidade Federal de Minas Gerais - UFMG - Instituto de Geociências, Departamento de Geografia. Av. Antônio Carlos, 6627 - 31270-901 - Belo Horizonte-MG, Brasil ribasgeo@gmail.com 


\section{1- INTRODUÇÃO}

A percepção da diminuição da qualidade ambiental pela sociedade, seja por meio da mídia, de informações científicas geradas por entidades governamentais, por empresas privadas ou do terceiro setor, vem alimentando discussões a respeito do pagamento por serviços ambientais. Serviços esses, que vem sendo há tempos prestados com gratuidade e por observação do cenário atual entende-se a necessidade de um retorno financeiro a quem se comprometa com a sua conservação em detrimento de apenas almejar o ganho econômico com a utilização de determinado recurso natural. O pagamentopelos serviços ambientais pode trazer à racionalidade de curto prazo usualmente adotada nas ações humanas, a racionalidade de longo prazo, mais desejável nas questões ambientais (FEARNSIDE, 1997).

A constituição federal de 1988 prevê que 25\% do valor arrecado pelo Imposto sobre Operações relativas à Circulação de Mercadorias e sobre Prestações de Serviços de Transporte Interestadual e Intermunicipal e de Comunicação (ICMS) seja repassado aos municípios. Dentre esse montante direcionado aos municípios, 1\% deve ser direcionado à retribuição por serviços ambientais realizados por estes. Essa distribuição deve ocorrer segundo à observação de índices relacionados à qualidade ambiental. Este modelo de repasse de recursos é conhecido como o ICMS Ecológico.

Atualmente no estado de Minas Gerais, a Lei Estadual no 18.030/2009, dispõe sobre os critérios para a distribuição do ICMS ecológico. Segundo esta Lei 1,1 \% do ICMS é repassado aos municípios em função do Índice de Meio Ambiente (IMA). O IMA é composto por três subcritérios, ponderados pelos respectivos pesos, a saber: Índice de Conservação (IC), com peso de 45,45\%, referente às Unidades de Conservação e outras áreas protegidas; Índice de Saneamento Ambiental (ISA) com peso de 45,45\%, referente aos aterros sanitários, estações de tratamento de esgotos e usinas de compostagem e, mais recentemente, o Índice de Mata Seca (IMS), com peso de 9,1\%, referente à presença e proporção em área da fitofisionomia Mata Seca no município.

O Instituto Estadual de Florestas (IEF) é o órgão responsável pelo IC e pelo IMS. Para determinação do IC são levados em consideração a área da unidade de conservação (UC) e/ou área protegida, a área do município e também parâmetros relacionados a um fator de conservação e de qualidade, que recebem notas a partir de avaliação anual realizada pelos responsáveis pelas UC's. Tais procedimentos para o cadastramento das UC's estão dispostos na Deliberação Normativa COPAM nº 86 de 17/07/2005 e Resolução da Secretaria Estadual de Meio Ambiente 
e Desenvolvimento Sustentável - SEMAD n 318/2005. O IMS considera a área de mata seca existente no município, cujo valor encontra-se disponível na versão mais atual do Inventário Florestal de Minas Gerais que possui duas vertentes: I) Mapeamento e monitoramento periódico da cobertura florestal natural das florestas produtivas do território mineiro; II) Inventário Florestal, propriamente dito, que gera uma série de informações em relação às florestas naturais, inclusive relacionadas à determinação do estoque de carbono e ao acompanhamento contínuo do desenvolvimento das florestas, por meio de medições em parcelas permanentes estabelecidas nas fitofisionomias florestais presentes no estado de Minas Gerais (SEMAD, 2014).

Já o ISA é analisado pela Fundação Estadual de Meio Ambiente (FEAM), baseando-se no número total de sistemas habilitados, tipo de empreendimento e porcentagem da população atendida no município. Estão aptos a receber o ICMS Ecológico, subcritério Saneamento, os municípios que possuem sistema de tratamento ou disposição final de lixo ou de esgoto sanitário, com operação licenciada ou autorizada pelo órgão ambiental estadual, que atendam, no mínimo, a, respectivamente, $70 \%$ e 50\% da população urbana. A Resolução Conjunta SEMAD-SEPLAG $\mathrm{n}^{\mathrm{o}}$ 1.212/2010 define os procedimentos para cálculos e publicação dos índices municipais e a Resolução SEMAD $n^{\circ}$ 1.273/2011 complementa a Resolução Conjunta 1.212/2010, estabelecendo os critérios e procedimentos para o cálculo do fator de qualidade de empreendimentos de tratamento e/ou disposição final de resíduos sólidos urbanos e de tratamento de esgotos sanitários a serem aplicados na distribuição. Ao final das análises dos 3 índices, a SEMAD é responsável pela compilação, publicação e consolidação do Índice de Meio Ambiente a partir dos dados fornecidos pela FEAM e pelo IEF, segundo o seguinte critério: $\mathrm{IMA}=0,4545 * \mathrm{ISA}+0,4545 * \mathrm{IC}+0,091 * \mathrm{IMS}$. Posteriormente, as informações são encaminhadas à Fundação João Pinheiro para o devido repasse aos municípios, que é realizado sempre no segundo dia útil da semana, sendo que o primeiro repasse do mês é feito com base no índice calculado no mês anterior (SEMAD, 2014).

A agricultura, enquanto uma atividade indispensável em termos de desenvolvimento regional e produção de alimentos, acarreta diversos impactos ambientais positivos e negativos, a partir de seu principal aspecto ambiental que é a modificação da forma de uso e ocupação do solo. Sob o ponto de vista positivo podemos dar destaque ao próprio incentivo ao crescimento econômico proporcionado pela atividade e sob o ponto de vista negativo podemos citar o desmatamento, tendo em vista a retirada da cobertura vegetal para ceder local a produção, ocasionando a perda de biodiversidade. A contaminação do solo e do lençol freático por defensivos e estimulantes agrícolas, devido a necessidade de fertilização da terra para a 
continuidade da colheita e da proteção destas contra pragas, entre outros prejuízos ambientais. Porém, de acordo com Guerin e Isernhagen (2013) atualmente a conciliação da produção agropecuária com a conservação ambiental já não é assunto só de produtores inovadores ou ambientalistas deslumbrados, visto a já existência de possibilidades técnicas e economicamente viáveis da produção rural ocorrer sem causar danos ambientais.

Tendo em vista o contexto apresentado, o objetivo do presente estudo é a segmentação dos municípios do Estado de Minas Gerais que receberam o ICMS Ecológico no ano de 2010 em função da sua produção agrícola no mesmo. Com isso pretende-se realizar uma análise dos agrupamentos formados por meio da técnica Two Step Cluster, oferecendo subsídio para o entendimento da configuração espacial do pagamento por serviços ambientais em Minas Gerais e o seu relacionamento com a atividade agrícola.

\section{2- PROCEDIMENTO METODOLÓGICO}

A análise de cluster é uma técnica exploratória da análise multivariada que busca agrupar elementos de dados baseando-se na similaridade entre eles, obtendo assim homogeneidade dentro dos grupos e heterogeneidade entre eles (FRALEY e RAFTERRY, 1998). A técnica Two Step Cluster é um algoritmo desenvolvido para desenvolver análises em bases de dados muito extensas e também é capaz de trabalhar com variáveis qualitativas e quantitativas simultaneamente. De acordo com Zhang et al. (1996) a técnica Two Step Cluster possui algumas especificidades que a diferenciam das demais técnicas de cluster, tais quais a capacidade de manipulação de variáveis contínuas e categóricas e a determinação automática do número de clusters.

A determinação da quantidade de clusters ocorre de acordo om algum critério de informação para a seleção dos melhores modelos. De acordo com Emiliano (2009) estes critérios penalizam a verossimilhança, utilizando o número de variáveis do modelo e, eventualmente o tamanho da amostra. Este procedimento é realizado subtraindo-se do valor da verossimilhança uma determinada quantidade, que depende do quão complexo é o modelo (quanto mais parâmetros, mais complexo).

Emiliano (2009) afirma que o critério de Informação de Akaike (AIC):

Desenvolvido por Hirotugu Akaike sob o nome de "um critério de informação" em 1971 e proposto, em Akaike (1974), é uma medida relativa da qualidade de ajuste de um modelo estatístico estimado. Fundamenta-se no conceito de entropia, oferecendo uma medida relativa das informações perdidas, quando um determinado modelo é usado para descrever a realidade.

Cadernos do Leste 
Após a análise de cluster o valor de entropia permite avaliar a qualidade do modelo, sendo que os valores menores são mais positivos para a análise e inversamente, os valores mais altos indicam um agrupamento de pior qualidade (SHIH et al., 2010). O AIC é rotineiramente utilizado como critério para a seleção de modelos, sendo que, o melhor modelo será aquele que apresentar o menor valor do critério. O AIC é um critério que avalia a qualidade do ajuste do modelo paramétrico estimado pelo método da máxima verossimilhança, definido como sendo, AIC $=-2$ (Função suporte maximizada) + 2 (número de parâmetros), tal qual a equação 1:

\section{$A A A A A A=-2 \log L L(\theta \theta)+2(k k)$ Equação 1}

A rodada do Two Step Cluster foi realizada no software SPSS. Para buscar a similaridade e fornecer o agrupamento foi utilizada a distância euclidiana e para a determinação do melhor modelo foi utilizado o Critério de Informação Akaike. Foram utilizados dados referentes ao valor de repasse de ICMS Ecológico aos municípios do Estado de Minas Gerais e valores de produção de lavouras temporárias e permanentes. Também foram utilizados o total em hectares das áreas plantadas e colhidas nos municípios do Estado no ano de 2010. Os valores totais em reais (R\$) de ICMS Ecológico repassado aos municípios de Minas Gerais no ano de 2010 foram obtidos no site da Fundação João Pinheiro (FJP, 2014). Os valores em reais (R\$) de produção de lavouras temporárias e permanentes e o total das áreas plantadas e colhidas são provenientes do Instituto Brasileiro de Geografia e Estatística (IBGE) e foram obtidos no site do Instituto de Pesquisa Econômica Aplicada (IPEA) (IPEADATA, 2014).

\section{3- RESULTADOS}

Conforme apresentado na Figura 1, foram definidos três clusters por meio do algoritmo Two Step Cluster, sendo também indicada uma boa qualidade da classificação destes clusters. 


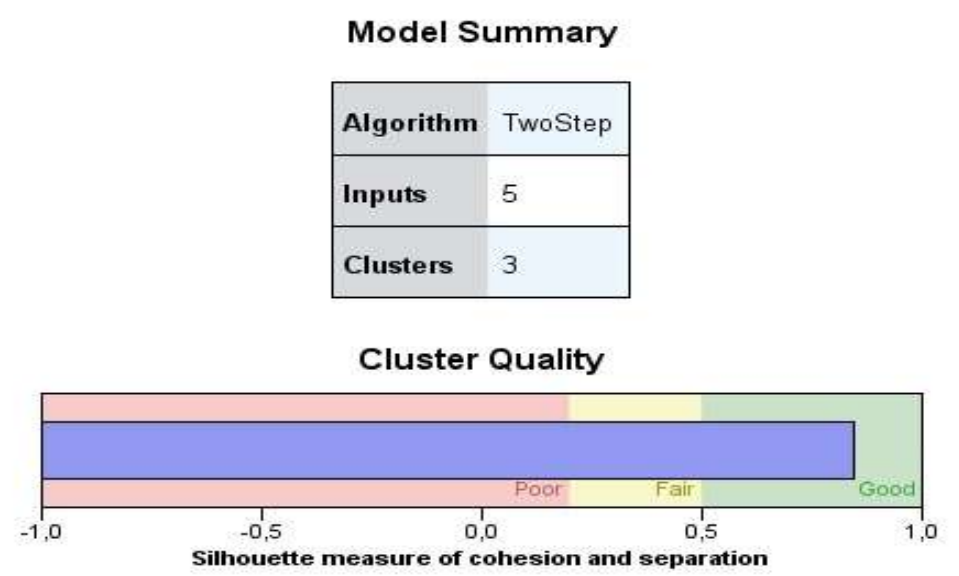

Figura 1:Clusters identificados e qualidade da classificação

$\mathrm{Na}$ tabela 1 apresentada a seguir estão dispostos os resultados obtidos na análise de cluster assim como a medidas de tendência central (média) e de variabilidade (desvio padrão) para cada variável incluída no modelo.

Tabela 1: Resultados obtidos na análise Two Step Cluster

\begin{tabular}{c|c|c|c|c|c|c}
\hline \multicolumn{2}{c|}{$\begin{array}{c}\text { Cluster / } \\
\text { Estatística }\end{array}$} & $\begin{array}{c}\text { ICMS } \\
\text { Ecológico } \\
(\mathbf{R} \$)\end{array}$ & $\begin{array}{c}\text { Área } \\
\text { Colheita } \\
(\text { hec) }\end{array}$ & $\begin{array}{c}\text { Área } \\
\text { Plantada } \\
(\text { hec) }\end{array}$ & $\begin{array}{c}\text { Lavoura } \\
\text { Permanente (R\$) }\end{array}$ & $\begin{array}{c}\text { Lavoura } \\
\text { Temporária (R\$) }\end{array}$ \\
\hline 1 & Média & 95.884 & 40.237 & 40.839 & 28.069 & 42.504 \\
\hline & N & 60 & 60 & 60 & 60 & 60 \\
\hline & Desvio & 208.135 & 36.266 & 35.729 & 24.760 & 46.214 \\
\hline $\mathbf{2}$ & Média & 0 & 3.176 & 3.214 & 3.018 & 2.807 \\
\hline & N & 305 & 305 & 305 & 305 & 305 \\
\hline & Desvio & 0 & 4.015 & 4.136 & 5.535 & 4.865 \\
\hline 3 & Média & 101.588 & 2.888 & 2.896 & 2.380 & 2.308 \\
\hline & N & 488 & 488 & 488 & 488 & 488 \\
\hline & Desvio & 182.841 & 3.466 & 3.506 & 4.793 & 4.352 \\
\hline Total & Média & 64.862 & 5.618 & 5.679 & 4.416 & 5.314 \\
\hline & N & 853 & 853 & 853 & 853 & 853 \\
\hline & Desvio & 156.577 & 13.994 & 14.018 & 10.471 & 16.560 \\
\hline
\end{tabular}

O agrupamento de número 1, formado por 60 municípios, recebe repasses de ICMS ecológico em valores superiores à média total observada e possui valores de área e produção agrícola bastante superiores à média total observada. São municípios que se caracterizam como grandes produtores agrícolas e mesmo diante deste fato, recebem maiores valores de repasse do 
ICMS Ecológico. O agrupamento de número 2, formado por 305 municípios, caracteriza-se por não receber repasses do ICMS Ecológico e por possuir os parâmetros relacionados a agricultura com valores abaixo da média, ou seja, são municípios com baixa produção agrícola. $O$ agrupamento de número 3, formado por 488 municípios, possui valores de repasses de ICMS Ecológico acima da média, porém os valores de área e produção agrícola estão abaixo da média observada.

Os resultados obtidos estão representados no mapa da Figura 2, que apresenta cada um dos clusters identificados em relação ao enquadramento das mesorregiões do Estado de Minas Gerais.

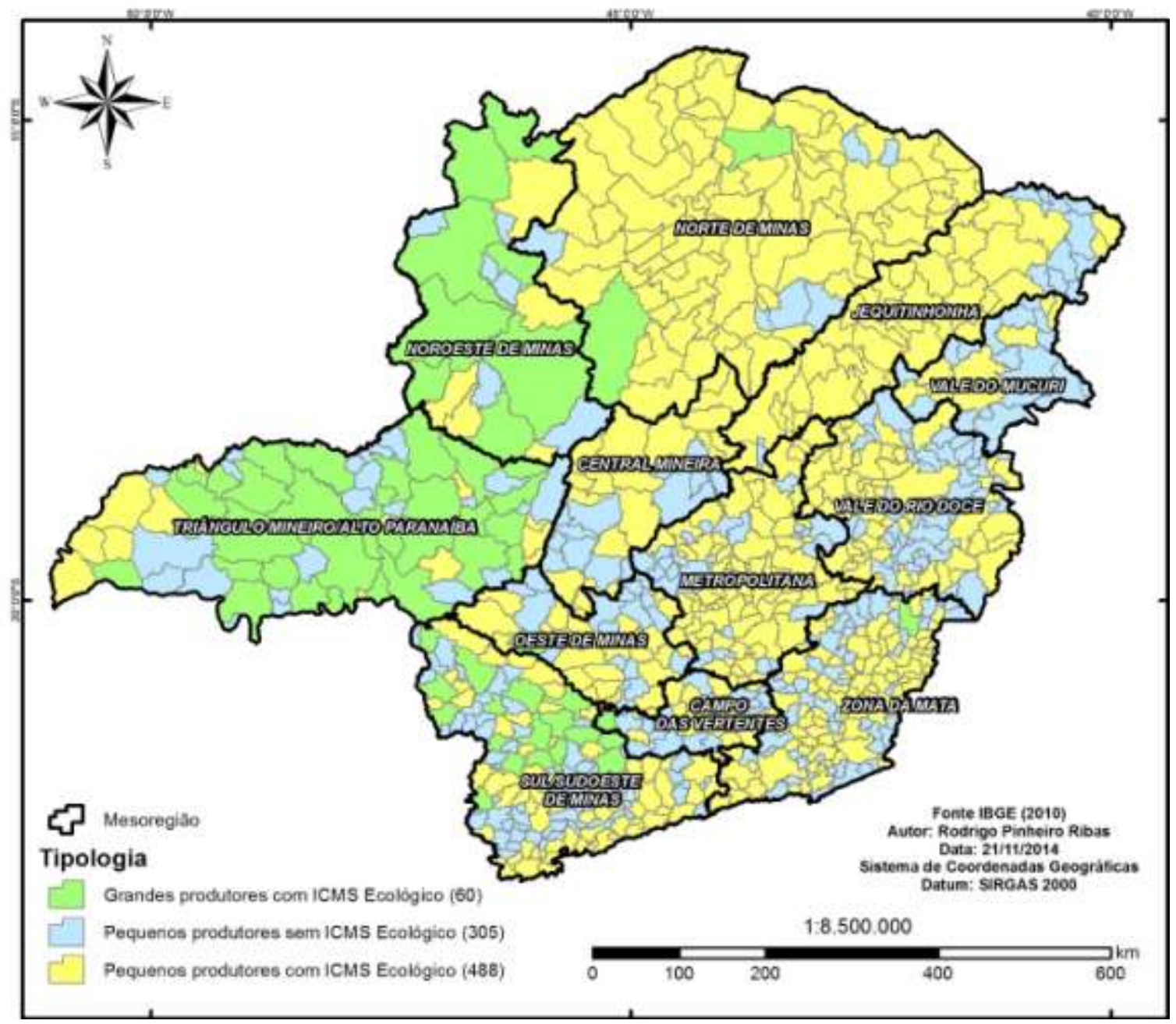

Figura 2: Distribuição regional dos clusters identificados

Analisando o gráfico box plot é possível uma melhor análise da distribuição dos dados e a identificação de outliers. No gráfico apresentado na Figura 3, configurando a formação dos clusters em relação ao repasse do ICMS Ecológico, os outliers do cluster 1, referentes à tipologia 
(Grandes produtores com ICMS Ecológico) é formado por municípios com receita de ICMS Ecológico com valores muito acima da média, com destaque para os municípios de

Uberaba e Uberlândia (indicados com os números 823 e 824, respectivamente no gráfico da Figura 3) que receberam em 2010 a quantia de $\mathrm{R} \$$ 813.427,00 e $\mathrm{R} \$ \mathbf{1 . 2 2 5 . 4 0 0 , 0 0}$ respectivamente. Os outliers do cluster 3 , referentes à tipologia (Pequenos produtores com ICMS Ecológico) ocorreram em grande número nestas observações e um padrão observado foram municípios com atividade agrícola quase inexistente, porém com valores de recebimento de ICMS Ecológico muito acima da média, com destaque para o município de Marliéria na região do Vale do Rio Doce, recebendo a quantia de R\$1.095.190,00 no ano de 2010.

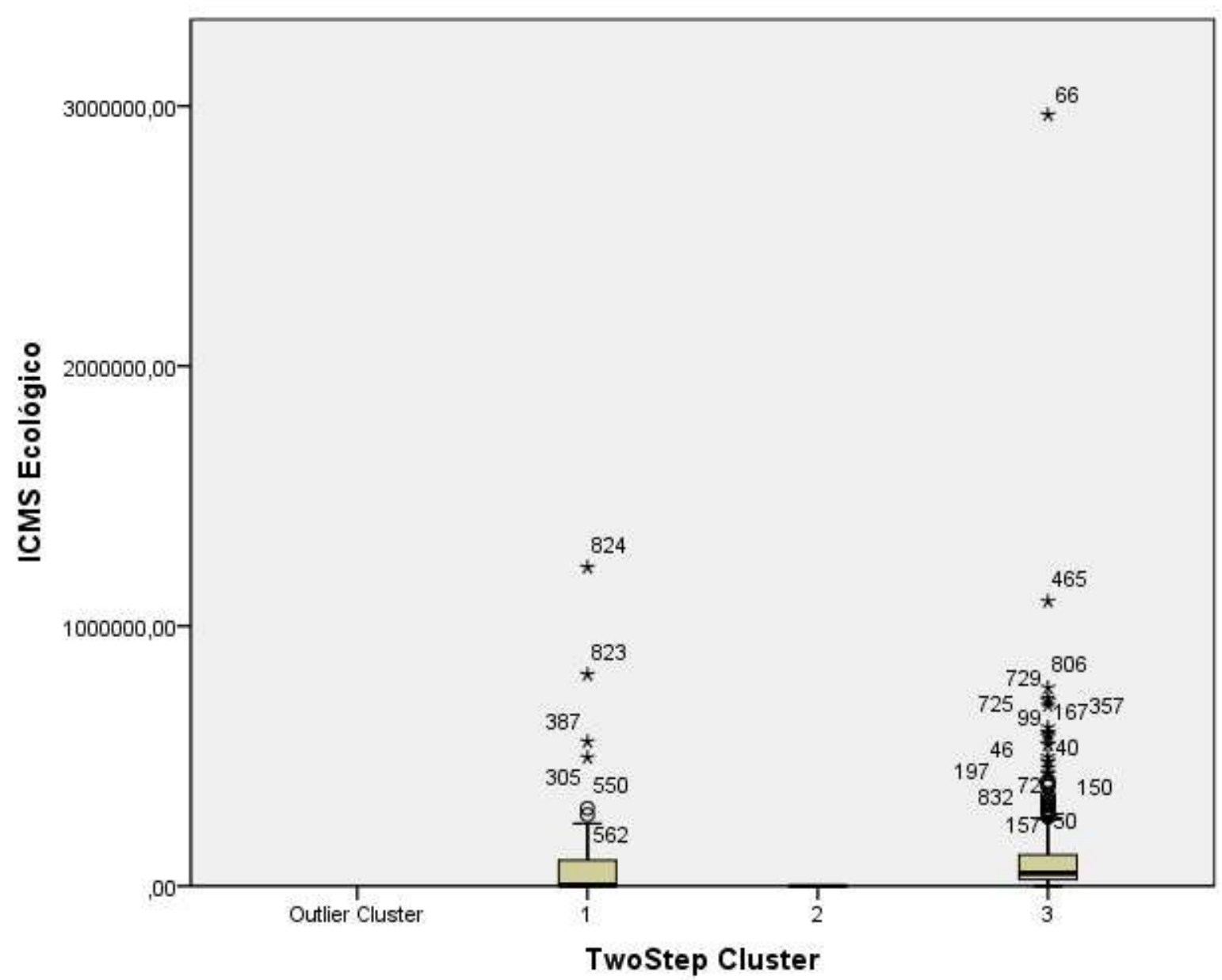

Figura 3:Box Plot - ICMS Ecológico

No gráfico apresentado na Figura 4, configurando a formação dos clusters em relação à área plantada total, os outliers do cluster 1, referentes à tipologia (Grandes produtores com ICMS Ecológico) é formado por municípios com área plantada total significativamente maior que os demais, e nesta mesma lógica, possuindo área de colheita e receita de produção das lavouras com 
valores muito acima da média. Destacam-se neste contexto os municípios de Uberaba e Unaí (indicados com os números 823 e 826, respectivamente no gráfico da Figura 4) que possuíam em 2010 área plantada total de 183.615 e 201.350 hectares respectivamente.

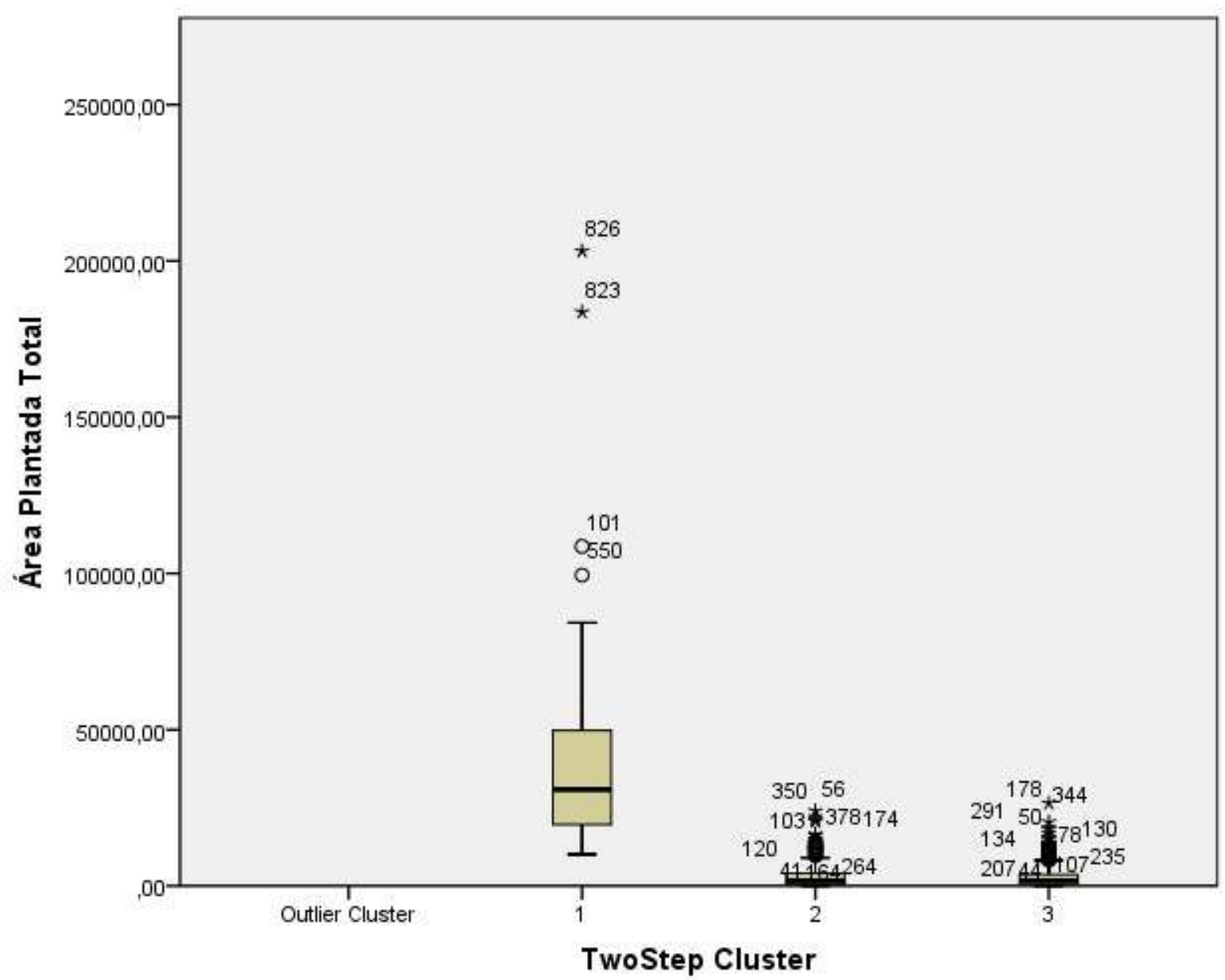

Figura 4:Box Plot-Área Plantada Total

\section{4- DISCUSSÕES E CONCLUSÃO}

Os resultados em questão permitem inferências acerca da produtividade agrícola no Estado, que possui maior concentração nas regiões do Triângulo Mineiro / Alto Paranaíba e Noroeste de Minas. Nas regiões Norte de Minas e Sul / Sudoeste de Minas notam-se alguns municípios pulverizados dentre os demais, possuindo expressiva produtividade agrícola e na região da Zona da Mata o município de Manhuaçu, grande produtor de café, se manteve no cluster com municípios que recebem valores de ICMS acima da média e também possuem a produtividade agrícola acima da média. Com relação ao pagamento por serviços ambientais é interessante notar que nestas regiões, o repasse do ICMS Ecológico possui em média as mais 
altas taxas do Estado. Conforme relatório da FEAM com panorama da destinação dos resíduos sólidos em Minas Gerais (FEAM, 2013) um fato que pode estar contribuindo para este cenário e a situação satisfatória e constante melhora da disposição final de resíduos sólidos observada na região do Triângulo Mineiro / Alto Paranaíba, com grande número de aterros sanitários regularizados.

É interessante perceber que no Estado de Minas Gerais o repasse do ICMS Ecológico não está necessariamente atrelado à preservação e proteção de áreas naturais, existindo também compensação por outras formas de manutenção da qualidade ambiental, tal qual o investimento no sistema de saneamento básico. Com isso ocorrem casos específicos como o de Uberlândia, município com poucas unidades de conservação, possuindo em sua maioria Parques Municipais e pequenas Reservas Particulares do Patrimônio Cultural (RPPN), sendo que apenas recentemente, no ano de 2014, foi aberta a primeira UC de Proteção Integral no município, o Parque Estadual do Pau Furado (IEF, 2014), porém, mesmo diante desta situação, é o segundo $\left(2^{\circ}\right)$ município que mais recebe o ICMS Ecológico em todo o Estado. Situação está permitida pelo fato de Uberlândia possuir $100 \%$ de água tratada e $99 \%$ de tratamento de esgoto no perímetro urbano, sendo eleita pela segunda vez consecutiva, a primeira $\left(1^{\mathrm{a}}\right)$ cidade mais bem colocada no ranking de saneamento no país (INSTITUTO TRATA BRASIL, 2014).

O perfil da região Norte e do Jequitinhonha apresenta a grande maioria dos municípios inseridos no cluster 3, possuindo valores de repasses de ICMS Ecológico acima da média, porém com valores de área e produção agrícola estando abaixo da média observada. Um grande indicador desta situação pode ser a grande presença da fitofisionomia Mata Seca nesta região, viabilizando um IMA mais alto em função do IMS. Outro aspecto que indica que a preservação de áreas protegidas também tem grande peso na forma de cálculo do montante de repasse de ICMS Ecológico no Estado é caso do município de Marliéria na região do Vale do Rio Doce, que possui grande parte do território inserido no Parque Estadual do Rio Doce, que representa um exemplo de qualidade de gestão numa área de cerca de 36.000 hectares, sendo uma das principais reservas de Mata Atlântica Contínua do Estado.

Conclui-se então que o exemplo do Estado de Minas Gerais na distribuição do ICMS Ecológico tem se mostrado promissor e relativamente equalizado, visto que consegue atingir um leque maior de munícipios, que de alguma forma direcionam ações em prol do meio ambiente. 


\section{REFERÊNCIAS BIBLIOGRÁFICAS}

EMILIANO, P.C. Fundamentos e aplicações dos critérios de informação: Akaike e Bayesiano. Dissertação de mestrado. Lavras: UFLA, 2009, 92 p.

FEARNSIDE, P.M. Environmental services as a strategy for sustainable development in rural Amazonia. Ecological Economics, v. 20, p. 53-70, 1997.

FRALEY, C.; RAFTERRY, A. E. How Many Clusters? Which Clustering Method? Answers via Model-based Cluster Analysis. Computer Journal, v.4, p. 578-588, 1998.

FUNDAÇÃO ESTADUAL DO MEIO AMBIENTE. Panorama da destinação dos resíduos sólidos urbanos no Estado de Minas Gerais em 2012. Belo Horizonte: FEAM, 2013, 39 p.

FUNDAÇÃO JOÃO PINHEIRO. FJP. Disponível em < http://www.fjp.mg.gov.br>. Acesso em: 23 outubro. 2014.

INSTITUTO DE PESQUISA ECONÔMICA APLICADA. IPEADATA. Disponível em www.ipeadata.gov.br. Acesso em: 23 outubro. 2014.

INSTITUTO ESTADUAL DE FLORESTAS. IEF. Disponível em http://www.ief.mg.gov.br/noticias/1/1893-parque-estadual-do-pau-furado-e-aberto-paravisitacao. Acesso em 25 novembro. 2014.

INSTITUTO TRATA BRASIL. Disponível em <http://www.tratabrasil.org.br/ranking-dosaneamento>. Acesso em 25 novembro. 2014.

NATALIA G., INGO I. Plantar, criar e conservar: unindo produtividade e meio ambiente. São Paulo: Instituto Socioambiental, 2013. ISBN 978-85-8226-015-9.

SECRETARIA ESTADUAL DE MEIO AMBIENTE E DESENVOLVIMENTO SUSTENTÁVEL. SEMAD.

Disponível em <http://www.semad.mg.gov.br/icms-ecologico>. Acesso em: 13 novembro. 2014.

SHIH, MING-YI; JHENG, JAR-WEN \& LAI, LIEN-FU. A two-step method for clustering mixed categorical and numerical data. Tamkang Journal of Science and Engineering, Vol. 13 n 1, pp. 11-19. 2010.

ZHANG, T., RAMAKRISHNAN, R., BIRCH, M. L. An efficient data clustering method for very large databases. In: Proceedings of the ACM SIGMOD Conference on Management of Data. Montreal, Canada: ACM. p. 103-114, 1996. 